\title{
Parallel hybrid enhanced inherited GA based SCUC in a distributed cluster
}

\author{
C. Christopher Columbus, Sishaj P. Simon \\ National Institute of Technology, Tiruchirappalli, Tamilnadu, India
}

Correspondence: C. Christopher Columbus. Address: National Institute of Technology, Tiruchirappalli, Tamilnadu, India. Telephone: 910-431-251-3298. Email: christoccc@gmail.com.

Received: April 4, 2012

DOI : 10.5430/air.v1n1p96
Published: September 1, 2012

URL: http://dx.doi.org/10.5430/air.v1n1p96

\section{Abstract}

In the deregulated electricity market, secure operation is an enduring concern of the independent system operator (ISO). For a secure and economical hourly generation schedule of the day ahead market, ISO executes the security constrained unit commitment (SCUC) problem. In this paper, a new formulation of SCUC problem, considering more practical constraints are presented. The proposed SCUC formulation includes constraints, such as hourly power demand, system reserves, ramp up/down limits, minimum ON/OFF duration limits. Unlike the traditional SCUC techniques the proposed method solves the Security Constrained Economic Dispatch (SCED) from the UC. To solve such SCUC model, a hybrid solution method consists of an enhanced inherited genetic algorithm (EIGA) is used for unit commitment master problem and Lambda relaxation method is used for the economic dispatch sub-problem. The message passing interface (MPI) based technique is used to implement the hybrid EIGA in distributed memory model. The time complexity and the solution quality with respect to the number of processors in a cluster are thoroughly analyzed. The effectiveness of the proposed method to solve the SCUC problem is shown on different test systems.

\section{Key words}

Enhanced inherited genetic algorithm, Security constrained unit commitment, Parallel computing, Distributed cluster, Security constrained economic dispatch

\section{I ntroduction}

Unit commitment (UC) plays a major role in the daily operation planning of power systems. System operators need to perform many UC studies, in order to economically assess the spinning reserve capacity required to operate the system as secure as possible. The objective of the UC problem is the minimization of the total operating cost of the generating units during the scheduling horizon, subject to system and unit constraints. The solution of the above problem is a very complicated procedure, since it implies the simultaneous solution of two sub problems: the mixed-integer nonlinear programming problem of determining the ON/OFF state of the generating units for every hour of the dispatch period and the quadratic programming problem of dispatching the forecasted load among them. However, the UC schedules are always unfeasible because of the network security constraints, such as the available of transmission capacity, are not taken into account in the traditional UC problems. Therefore the security constrained unit commitment (SCUC), which actually 
can provide a financially viable UC, that is physically feasible, is considered to be one of the best available options in power system operation ${ }^{[1,2]}$.

The security analysis of the power system is the process of finding whether the existing system is in secure state or not. When the load is satisfied and no violations in the limits due to uncertainties, then the system is in the secure state. If the limits are violated or the loads are not meeting the demands then the system is in insecure state.

Power system security problems are classified as static and dynamic ${ }^{[3]}$. Evaluating the power system problem without considering the transient and time dependent variations is termed as static security problem. There are so many methods are executed to solve static problems. The dynamic problems are solved by the methods like priority list method ${ }^{[4]}$, dynamic programming methods ${ }^{[5,6]}$, branch and bound methods, Benders partitioning methods and Lagrangian relaxation (LR) methods ${ }^{[7-10]}$. The LR approaches are characterized by their ability to handle various constraints and to estimate the optimality of the solution in practical applications. The Lagrangian dual function is formed by adjoining a set of coupling constraints to the unit commitment primal objective function via Lagrange multipliers. The dual problem is decoupled into unit based sub-problems which are easier to solve. Due to the non convexity of the resource scheduling problem, difficulties were reported in obtaining a feasible solution. This paper solves the SCUC problem with line flow constraints through effective application of parallel hybrid EIGA in a distributed cluster. The constraints such as minimum up/down time, ramp up/down constraints, system transmission losses, and power balance equation as an equality constraint, limits on the active power generations of the units and limits on the currents in different lines as the inequality constraints are considered. The performance of the parallel hybrid EIGA in terms of solution quality is compared with that of other algorithms reported in literature.

This paper is organized as follows: Section 2 gives the problem formulation. Setting up of distributed cluster model is presented in section 3 . The theory of enhanced inherited GA is presented in section 4 . The simulation results are discussed in section 5 and the conclusion is in Section 6.

\section{Problem formulation}

The objective of SCUC is minimizing the fuel cost simultaneously satisfying equality and inequality constraints with security and transmission network constraints. The objective function of the SCUC can be formulated as follows:

Minimize

$$
\sum_{t=1}^{T} \sum_{i=1}^{N}\left[F_{i}\left(P_{(i, t)}\right) * I_{(i, t)}+S U_{i}^{t} * I_{(i, t)} *\left(1-I_{(i, t-1)}\right)+S D_{i}^{t} *\left(1-I_{(i, t)}\right)\right]
$$

where $\mathrm{i}$ - unit index (generator $i=1 . . \mathrm{N})$, N- number of units, $t$ - hour index $(t=1 \ldots \mathrm{T}), P_{(i, t)}$-active power output of unit $\mathrm{i}$ at time $t(\mathrm{MW}), I_{(i, t)}$ - commitment state of unit $i$ at time $t(1=\mathrm{ON}$ and $0=\mathrm{OFF}), S U_{i}^{t}, S D_{i}^{t}$ are the startup cost and shut down cost of unit $i$ at time $t$, respectively.

$$
F_{i}\left(P_{(i, t)}\right)=a+b * P_{(i, t)}+c^{*} P_{(i, t)}^{2}
$$

where, $F_{i}\left(P_{(i, t)}\right)$ - Fuel cost function of $i$ th unit $(\$ / \mathrm{h}), a, b$ and $c$ are the fuel cost co-efficients.

Subject to the following constraints: 


\subsection{System constraints}

Power balance constraints

$$
\begin{array}{cc}
P_{G i, t}-P_{D i, t}=V_{i, t} \sum_{j=1}^{N} V_{j, t}\left(G_{i j} \cos \theta_{i j}+B_{i j} \sin \theta_{i j}\right) & i \in N_{B-1} \\
Q_{G i, t}-Q_{D i, t}=V_{i, t} \sum_{j=1}^{N} V_{j, t}\left(G_{i j} \sin \theta_{i j}-B_{i j} \cos \theta_{i j}\right) & i \in N_{P Q}
\end{array}
$$

where, $P_{G i, t}, Q_{G i, t}$ - The active and reactive power generation at bus i at time t, respectively. $P_{D i, t}, Q_{D i, t}$ - The active and reactive power demand at bus i at time t, respectively. $G_{i j}, B_{i j}$ - Conductance and susceptance between bus $i$ and bus $j$, respectively. $V_{i, t}$-Voltage magnitude of bus $i$ at time $t(\mathrm{pu}) . N_{B}$-Number of busses $N_{B-1}$ - Number of busses excluding slack bus, $N_{P Q}$ - Number of PQ buses. For this problem the full Newton-Raphson method is used for solving the AC power flow equations.

Spinning reserve constraints

$$
\sum_{i=1}^{N}\left(P_{i, \max } * I_{(i, t)}\right) \geq \operatorname{Load}_{t}, k \in[1, T]
$$

Where, $\mathrm{t}$ is the time index and $\mathrm{T}$ is the total number of hour.

\subsection{Unit constraints}

\subsubsection{Minimum up and down time constraints}

A unit must be ON/OFF for a minimum period before it can be shut off or brought online respectively.

$$
\begin{aligned}
& {\left[X^{\text {on }}(i, t-1)-T^{\text {on }}(i)\right] *\left[I_{(i, t-1)}-I_{(i, t)}\right] \geq 0} \\
& {\left[X^{\text {off }}(i, t-1)-T^{\text {off }}(i)\right] *\left[I_{(i, t)}-I_{(i, t-1)}\right] \geq 0}
\end{aligned}
$$

where $X^{\text {on }}(i t), X^{\text {off }}(i, t)$ - ON and OFF duration of unit $i$ at time $t$, respectively. $T^{\text {on }}(i), T^{\text {off }}(i)$ - Minimum up (MUT) and down (MDT) time of unit i, respectively.

\subsubsection{Unit ramp constraints}

Ramp rate limits reflect the time it takes to turn the generators on/off, while uptime and downtime constraints ensure that once a generator is up/down, it stays at that state for at least a given length of time.

$$
\begin{aligned}
& P_{(i, t)}-P_{(i, t-1)} \leq U R(i) \\
& P_{(i, t-1)}-P_{(i, t)} \leq D R(i)
\end{aligned}
$$

where, UR(i) - Ramp-up rate limit of $i^{\text {th }}$ generator unit, $D R(i)$ - Ramp-down rate limit of $i^{\text {th }}$ generator unit.

Security constraints 


$$
\begin{array}{ll}
V_{i}^{\min } \leq V_{i, t} \leq V_{i}^{\max } & i \in N_{B-1} \\
\left|B F_{i, t}\right| \leq B F_{i}^{\max } & i \in N_{B}
\end{array}
$$

where, $V_{i}^{\min }, V_{i}^{\max }$ - Minimum and maximum voltage magnitude limit at bus i (pu), respectively. $B F_{i, t}-$ Power flow through branch $i$ at time $t$ (MVA). $B F_{i}^{\max }$ - Maximum flow limits for branch i (MVA). These security constraints are related to steady state conditions and it should be considered in the post-contingency states.

Reactive power generation limits

$$
Q_{G i}^{\min } \leq Q_{G i, t} \leq Q_{G i}^{\max }
$$

where $Q_{G i}^{\min }, Q_{G i}^{\max }$-Minimum and maximum reactive power generation limit for unit i, respectively.

\section{Parallel clustering environment}

In clusters, powerful low cost workstations and/or PCs are linked through fast communication interfaces to achieve high performance parallel computing. Workstation clusters have become an increasingly popular alternative to traditional parallel supercomputers for many workloads requiring high performance computing. The use of parallel computing for scientific simulations has increased tremendously in the last ten years, and parallel implementations of scientific simulation codes are now in widespread use ${ }^{[11-13]}$. There are two dominant parallel hardware/software architectures in use today are Distributed memory, and Shared memory. In shared memory systems, parallel processing occurs through the use of shared data structures, or through emulation of message passing semantics in software. Distributed memory systems are composed of a number of interconnected computational nodes, which do not share memory, but can communicate with each other through a high-performance ether net switch (HPES) as shown in Figure 1. Parallelism is achieved on distributed memory systems with multiple copies of the parallel program running on different nodes, sending messages to each other to coordinate computations. The cluster should perform as a parallel computing resource, achieving higher performance than possible using workstations configured in a more standard way. The nodes in the cluster are always used in groups, not individually as in a general purpose workstation laboratory.

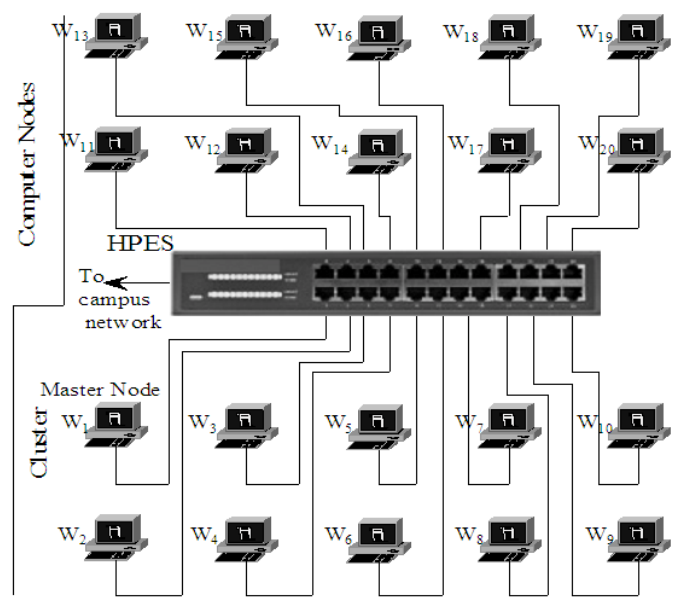

Figure 1. Distributed cluster of workstations (20 Nodes) 


\section{Speedup factor and efficiency}

To evaluate the parallel performance of the parallel hybrid EIGA, the speedup factor SWh and efficiency EWh of the cluster ${ }^{[14]}$ is calculated as follows;

$$
\begin{aligned}
& S W_{h}=W_{t} / W_{h t} \\
& E W_{h}=S W_{h} / W_{h}
\end{aligned}
$$

where $\mathrm{W}_{\mathrm{t}}$ and $\mathrm{W}_{\mathrm{ht}}$ are the execution time of single processor and cluster respectively.

\section{Overview of genetic algorithm}

GA is general purpose optimization algorithm based on the mechanics of natural selection and genetics ${ }^{[15-17]}$. They operate on string structures (chromosomes), typically a concatenated list of binary digits representing a coding of the control parameters (phenotype) of a given problem. Chromosomes themselves are composed of genes. The criterion which evaluates the quality of each chromosome is given by the Fitness corresponding to the evaluation of each individual for the objective function. Once the fitness of each of the individuals in the population is known, it is subjected to a Selection process in which the best evaluated individuals have a greater probability of being chosen as Parents for the exchange of genetic information called Crossover. Then a percentage of the Offspring's (individuals generated in the crossover) are subjected to the Mutation process in which a random change is generated in the chromosome. This mutation process provides greater diversity between the individuals in the population. When the crossover and mutation processes are complete a new population is generated which replaces the original population. This must be repeated until one of the convergence criteria defined for the problem is met. Each of these cycles is known as a Generation.

\subsection{Enhanced inherited crossover operation}

Crossover is an extremely important operator for the GA. It is responsible for the structure recombination (information exchange between mating chromosomes) and the convergence seed of the GA and is usually applied with high probability (0.6-0.9). The chromosomes of the two parents selected are combined to form new chromosomes that inherit segments of information stored in parent chromosomes.

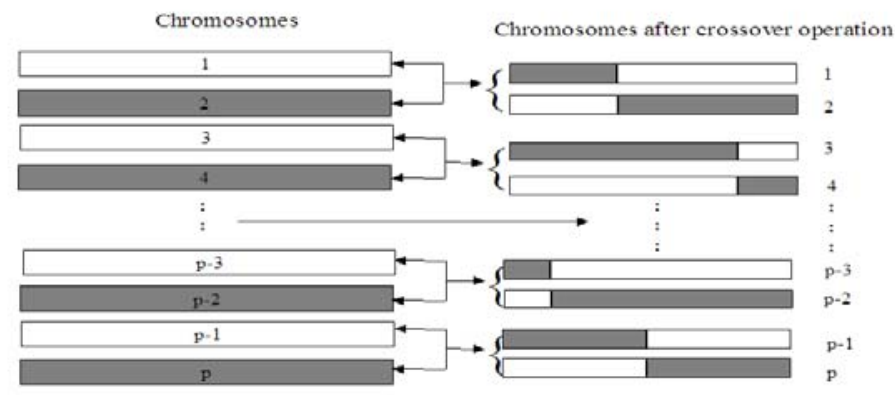

Figure 2. Traditional crossover operation

Traditionally, in GA a linear crossover is performed on the two chromosome represented as a string, as shown in Fig.2. Here a chromosome 1 is combined with chromosome 2 of randomly selected chromosomes and two new chromosomes are formed from the information stored in parent chromosomes. Similar new chromosomes are formed between 3 and 4 and so on. Here chance of inheriting information from parent chromosomes is less, since there may be chance to inherit more information while combining the chromosomes 2 and 3 instead of chromosomes 1 and 2. In order to avoid this 
disadvantage, an enhanced inherited crossover operation is carried to transfer more information from parent to chromosomes ${ }^{[18]}$.

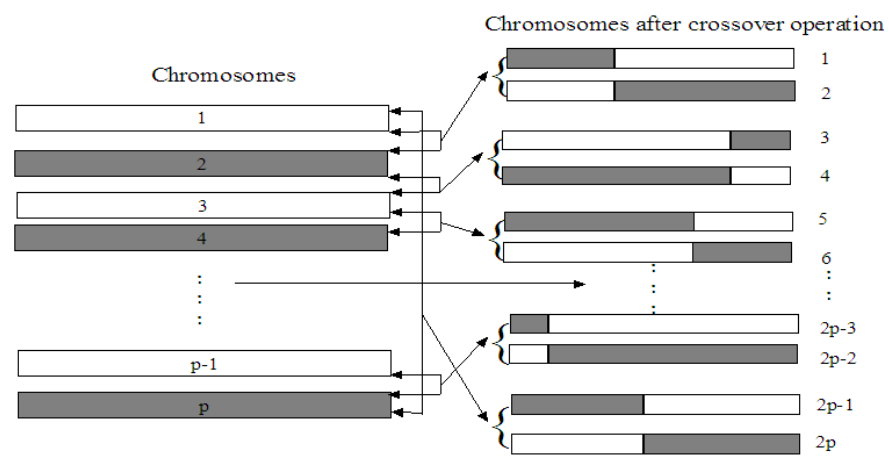

Figure 3. Enhanced inherited crossover operation

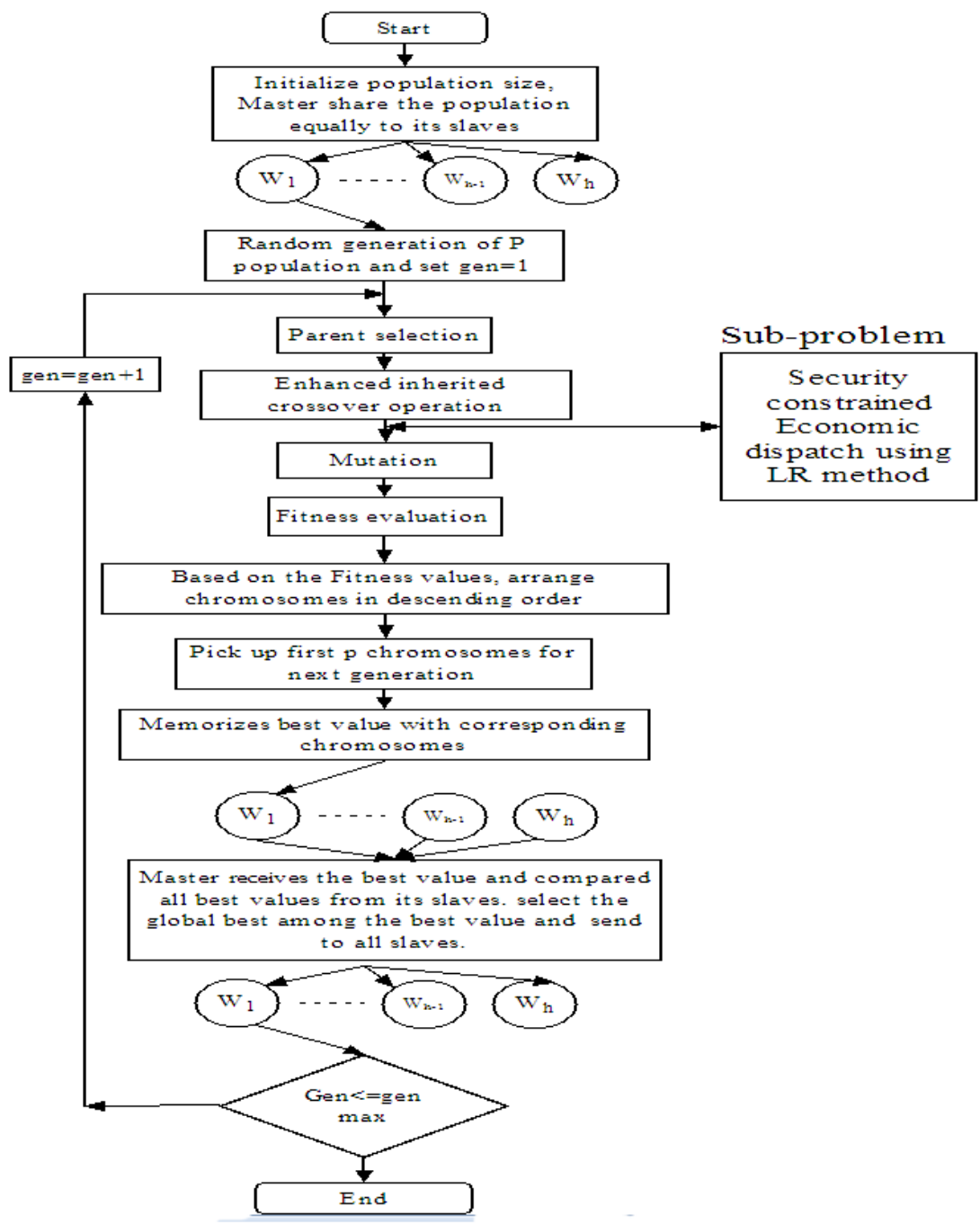

Figure 4. Flowchart for proposed enhanced inherited GA 
Figure 3 clearly shows the enhanced inherited crossover operation. At the end of the enhanced crossover operation, the number of population in GA is doubled, thereby the proposed enhanced inherited crossover operation inheriting more operation from the parent chromosomes. Then a percentage of the offspring (individuals generated in the crossover) are subjected to the Mutation process in which a random change is generated in the chromosome. This mutation process is carried for $2 p$ size population and provides greater diversity between the individuals in the population when compared to simple genetic algorithm.

When the crossover and mutation processes are complete, for new $2 \mathrm{p}$ size population, the fitness is evaluated for each individual for the objective function. Once the fitness of each of the individuals in the population is evaluated, then individuals are arranged in descending based on the fitness values. Then first p population is picked up for the next generation and process is repeated until it termination criteria is met. Here $\mathrm{p}$ is a population size. The flow of algorithm is shown in Figure 4.

The total population is shared among all slaves by using population sharing policy (PSP).

\subsection{Population sharing policy}

The master node decides the sharing of population by PSP. The population allocated in a slave processors or workers is given by

$$
\operatorname{Pop}_{P S P}=\left\{\begin{array}{l}
x, \text { if } N_{\text {pop }} \text { divisible by } W_{h} \\
(x+1) \text { for the first } h x \text { slaves and } x \text { for the remaining, Otherwise }
\end{array}\right.
$$

Where

$$
\begin{aligned}
& x=\text { floor }\left(N_{p o p} / W_{h}\right) \\
& h x=N_{p o p}-\left(x^{*} W_{h}\right)
\end{aligned}
$$

The master node allocates $(\mathrm{x}+1)$ population to the first hx slaves in the $W_{h} \operatorname{cluster}\left(W_{1} \ldots W_{h x} \ldots W_{h}\right)$ and $x$ population to the remaining slaves $\left(W_{h x+1} \ldots W_{h}\right)$.

\section{Results and discussions}

Simulations are carried out on a standard benchmark 6 bus system with 3 generators and a modified IEEE 30 bus system with 9 generators. All simulations are performed using a 20 node cluster on the MATLAB 7.5 environment. The cluster contains each computer nodes with Pentium(R) $3.40 \mathrm{GHz}$, 1GB RAM system.

\subsection{Case 1: Standard 6 bus System}

The proposed method has been applied to standard 6 bus system with 3 generators and 5 transmission lines and two tap changing transformers. The data is taken from the references ${ }^{[1,19]}$. The test system is validated for the traditional unit commitment and security constrained unit commitment. The problem has been solved at two phases, the UC has been solved by the parallel EIGA method as the maser problem and the economic dispatch with security constrained sub-problem by Lambda iterative method. The parameter settings for the EIGA as population= 50; crossover $=0.8$, mutation $=0.04$ and maximum number of iterations is 100 . The parameters are fixed by the trial and error method. 
The results obtained are compared with the existing literature. In this case, the economical unit G1 is fully committed and the expensive units G2 and G3 are not committed at certain hours in order to minimize the operating cost. The network constraints are checked and the constraints maintained within the limits. The daily operating cost is $\$ 83406.08$ for traditional UC and a daily operating cost of $\$ 84262.80$ for SCUC. In both cases the proposed parallel hybrid EIGA method overcomes the available method which is in the literature. The dispatch schedule of the six bus system for traditional UC and SCUC and its related costs are given in Table 1.

Table 1. Dispatch schedule of six bus system for traditional UC and SCUC

\begin{tabular}{|c|c|c|c|c|c|c|c|c|c|c|c|c|}
\hline \multirow[b]{2}{*}{ Hour } & \multicolumn{6}{|c|}{ UC-six - bus } & \multicolumn{6}{|c|}{ SCUC-six-bus } \\
\hline & $\begin{array}{l}\text { G1 } \\
\text { (MW) }\end{array}$ & $\begin{array}{l}\text { G2 } \\
\text { (MW) }\end{array}$ & $\begin{array}{l}\text { G3 } \\
\text { (MW) }\end{array}$ & $\begin{array}{l}\text { Fuel Cost } \\
\text { (\$) }\end{array}$ & $\begin{array}{l}\text { Start-up } \\
\text { Cost (\$) }\end{array}$ & $\begin{array}{l}\text { Total } \\
\text { Operating } \\
\text { Cost (\$) }\end{array}$ & $\begin{array}{l}\text { G1 } \\
\text { (MW) }\end{array}$ & $\begin{array}{l}\text { G2 } \\
\text { (MW) }\end{array}$ & $\begin{array}{l}\text { G3 } \\
\text { (MW) }\end{array}$ & $\begin{array}{l}\text { Fuel Cost } \\
\text { (\$) }\end{array}$ & $\begin{array}{l}\text { Start-up } \\
\text { Cost (\$) }\end{array}$ & $\begin{array}{l}\text { Total } \\
\text { Operating } \\
\text { Cost }(\$)\end{array}$ \\
\hline 1 & 178.69 & 10 & 0 & 3057.99 & 0 & 3057.99 & 169.34 & 10 & 0 & 2930.50 & 0 & 2930.50 \\
\hline 2 & 168.45 & 0 & 0 & 2462.33 & 0 & 2462.33 & 169.09 & 0 & 0 & 2471.10 & 0 & 2471.10 \\
\hline 3 & 161.84 & 0 & 0 & 2372.22 & 0 & 2372.22 & 162.43 & 0 & 0 & 2380.30 & 0 & 2380.30 \\
\hline 4 & 157.83 & 0 & 0 & 2317.57 & 0 & 2317.57 & 158.39 & 0 & 0 & 2325.20 & 0 & 2325.20 \\
\hline 5 & 158.16 & 0 & 0 & 2322.07 & 0 & 2322.07 & 158.72 & 0 & 0 & 2329.70 & 0 & 2329.70 \\
\hline 6 & 163.69 & 0 & 0 & 2397.43 & 0 & 2397.43 & 164.29 & 0 & 0 & 2405.60 & 0 & 2405.60 \\
\hline 7 & 176.86 & 0 & 0 & 2577.02 & 0 & 2577.02 & 177.56 & 0 & 0 & 2586.60 & 0 & 2586.60 \\
\hline 8 & 198.21 & 0 & 0 & 2868.45 & 0 & 2868.45 & 199.09 & 0 & 0 & 2880.50 & 0 & 2880.50 \\
\hline 9 & 199.67 & 10.00 & 0 & 3344.39 & 200 & 3544.39 & 200.54 & 10.00 & 0 & 3356.30 & 200 & 3556.30 \\
\hline 10 & 213.54 & 10.00 & 0 & 3533.93 & 0 & 3533.93 & 204.44 & 10.00 & 10.00 & 3723.10 & 0 & 3723.10 \\
\hline 11 & 213.18 & 10.00 & 10.00 & 3842.50 & 0 & 3842.5 & 213.72 & 10.44 & 10.00 & 3864.30 & 0 & 3864.30 \\
\hline 12 & 220.00 & 10.00 & 10.80 & 3949.86 & 0 & 3949.86 & 219.26 & 10.00 & 18.81 & 4080.90 & 0 & 4080.90 \\
\hline 13 & 220.00 & 10.00 & 17.03 & 4059.67 & 0 & 4059.67 & 220.00 & 10.50 & 17.58 & 4085.90 & 0 & 4085.90 \\
\hline 14 & 220.00 & 10.00 & 18.47 & 4085.07 & 0 & 4085.07 & 219.61 & 10.00 & 19.90 & 4105.00 & 0 & 4105.00 \\
\hline 15 & 220.00 & 13.83 & 20.00 & 4237.01 & 0 & 4237.01 & 219.60 & 15.62 & 20.00 & 4290.00 & 0 & 4290.00 \\
\hline 16 & 220.00 & 30.90 & 20.00 & 4794.25 & 0 & 4794.25 & 219.94 & 32.00 & 20.00 & 4829.40 & 0 & 4829.40 \\
\hline 17 & 220.00 & 50.12 & 20.00 & 5422.38 & 0 & 5422.38 & 219.66 & 51.50 & 20.00 & 5462.90 & 0 & 5462.90 \\
\hline 18 & 220.00 & 11.68 & 20.00 & 4166.86 & 0 & 4166.86 & 219.21 & 13.50 & 20.00 & 4215.40 & 0 & 4215.40 \\
\hline 19 & 220.00 & 10.89 & 20.00 & 4141.09 & 0 & 4141.09 & 218.41 & 13.50 & 20.00 & 4204.50 & 0 & 4204.50 \\
\hline 20 & 220.00 & 10.00 & 12.10 & 3972.76 & 0 & 3972.76 & 212.95 & 10.00 & 20.00 & 4015.70 & 0 & 4015.70 \\
\hline 21 & 220.00 & 10.00 & 12.05 & 3971.88 & 0 & 3971.88 & 219.59 & 10.00 & 13.50 & 4029.30 & 0 & 4029.30 \\
\hline 22 & 220.00 & 11.68 & 0 & 3677.06 & 0 & 3677.06 & 211.74 & 10.00 & 10.89 & 3835.00 & 0 & 3835.00 \\
\hline 23 & 198.07 & 0 & 0 & 2866.53 & 0 & 2866.53 & 198.95 & 0 & 0 & 2878.60 & 0 & 2878.60 \\
\hline 24 & 190.69 & 0 & 0 & 2765.76 & 0 & 2765.76 & 191.51 & 0 & 0 & 2777.00 & 0 & 2777.00 \\
\hline \multicolumn{6}{|c|}{ Total Cost } & 83406.08 & \multicolumn{5}{|c|}{ Total Cost } & 84262.80 \\
\hline
\end{tabular}

From the Table 1 itself it is understood that the UC schedule is not a feasible one for the practical operations. At hour 10 the line flow limit of line 1-4 is 100 MW only. It is violated in the UC schedule meanwhile it is corrected in the SCUC schedule by scheduling the third generator into ON condition. In this method all the voltage limits of the busses are generated and maintain between the ranges of 0.95 to 1.1. So the voltage violations are controlled. Table 2 shows the superiority of the proposed hybrid EIGA method with the other method, which is available in the literature.

Table 2. Comparison of results for six bus system

\begin{tabular}{lll}
\hline Problem & Method & Operating cost \\
\hline \multirow{2}{*}{ UC } & SDP ${ }^{[19]}$ & 83429.10 \\
& Parallel Hybrid EIGA & 83406.08 \\
\multirow{2}{*}{ SCUC } & SDP ${ }^{[19]}$ & 84268.70 \\
& Parallel Hybrid EIGA & 84262.80 \\
\hline
\end{tabular}


Even though the proposed hybrid model produces better results than the existing method, more execution time is required for simulation. In order to reduce the execution time the hybrid model is validated in a distributed cluster model with varying cluster sizes. It shows a tremendous reduction of computation time in the simulation.

Table 3 shows the execution time, speedup factor and cluster efficiencies achieved by the different cluster sizes for UC. When the cluster size increases the speedup factor also increases. i.e., performance of the cluster will increase. The time taken for sequential execution of the six bus 3 unit system is $128.42 \mathrm{sec}$ and it has been remarkably reduced to $13.77 \mathrm{sec}$, when it is executed by a 20 node cluster.

Table 3. Comparison of execution time, speedup factor and cluster efficiencies of six bus system for UC

\begin{tabular}{llllllllllll}
\hline No of Processors & $\mathbf{1}$ & $\mathbf{2}$ & $\mathbf{4}$ & $\mathbf{6}$ & $\mathbf{8}$ & $\mathbf{1 0}$ & $\mathbf{1 2}$ & $\mathbf{1 4}$ & $\mathbf{1 6}$ & $\mathbf{1 8}$ & $\mathbf{2 0}$ \\
\hline Execution time (s) & 128.42 & 81.52 & 50.99 & 37.42 & 28.26 & 23.48 & 20.32 & 17.97 & 15.86 & 14.52 & 13.77 \\
$\begin{array}{l}\text { Speedup factor } \\
\begin{array}{l}\text { Cluster efficiency } \\
\text { (\%) }\end{array}\end{array}$ & -- & 1.57 & 2.51 & 3.42 & 4.53 & 5.45 & 6.31 & 7.13 & 8.07 & 8.81 & 9.29 \\
\hline
\end{tabular}

Table 4 shows the execution time, speedup factor and cluster efficiencies achieved by the different cluster sizes for SCUC. When the cluster size increases the speedup factor also increases. i.e., performance of the cluster will increase. The time taken for sequential execution of the six bus system with 3 generators for SCUC is $1068.24 \mathrm{sec}$ and it has been remarkably reduced to $118.42 \mathrm{sec}$, when it is executed by a 20 node cluster.

Table 4. Comparison of execution time, speedup factor and cluster efficiencies of six bus system for SCUC

\begin{tabular}{llllllllllll}
\hline No of Processors & $\mathbf{1}$ & $\mathbf{2}$ & $\mathbf{4}$ & $\mathbf{6}$ & $\mathbf{8}$ & $\mathbf{1 0}$ & $\mathbf{1 2}$ & $\mathbf{1 4}$ & $\mathbf{1 6}$ & $\mathbf{1 8}$ & $\mathbf{2 0}$ \\
\hline Execution time (s) & 1068.24 & 693.51 & 428.91 & 313.19 & 238.39 & 199.62 & 170.61 & 151.92 & 136.57 & 124.04 & 118.42 \\
Speedup factor & -- & 1.54 & 2.49 & 3.41 & 4.48 & 5.35 & 6.26 & 7.03 & 7.82 & 8.68 & 9.02 \\
Cluster efficiency (\%) & -- & 77 & 62.25 & 56.83 & 56 & 53.5 & 52.26 & 50.21 & 48.87 & 48.22 & 45.1 \\
\hline
\end{tabular}

\subsection{Case 2: IEEE 30 bus System}

The proposed method has been applied to modified IEEE 30 bus system with 9 generators and 41 transmission lines. The data is taken from the reference ${ }^{[20]}$. The test system is validated for security constrained unit commitment. The parameter settings for the Case 1 are extended for this test system also.

The result obtained is compared with the existing literature. In this case, the economical units G4, G5, G6 and G7 are fully committed and the expensive units G8 and G9 are partially committed. Meanwhile the high expensive units G1, G2 and G3 are fully neglected to perform the economic operation. The network constraints are checked and the constraints are maintained within the limits. The daily operating cost of $\$ 151128.30$ for SCUC by the proposed hybrid EIGA model. In this case the proposed parallel hybrid EIGA method overcomes the available method, which is available in the literature. Ali Daneshi and et al. ${ }^{[20]}$ proposed a SCUC method in their Integration of Wind power and Energy Storage in SCUC problem paper and they recorded the operation cost as $\$ 151,119.09$. Meanwhile the SCUC problem solved using parallel hybrid EIGA method obtain a lesser operating cost of $\$ 151,113.20$. The dispatch schedule of the 30 bus system for SCUC and its related costs are given in Table 5.

Table 6 shows the execution time, speedup factor and cluster efficiencies achieved by the different cluster sizes for SCUC. When the cluster size increases the speedup factor also increases. i.e., performance of the cluster will increase, the time taken for sequential execution of the 30 bus 9 unit systems is $1562.34 \mathrm{sec}$ and it has been remarkably reduced to 141.74 sec, when it is executed by a 20 node cluster. 
Table 5. Dispatch schedule of IEEE 30 bus system for SCUC

\begin{tabular}{|c|c|c|c|c|c|c|c|c|c|c|c|c|}
\hline \multirow[t]{2}{*}{ Hour } & \multicolumn{9}{|c|}{ Dispatched Power in MW } & \multirow{2}{*}{$\begin{array}{l}\text { Fuel Cost } \\
\text { (\$) }\end{array}$} & \multirow{2}{*}{$\begin{array}{l}\text { Startup } \\
\text { Cost } \\
(\$)\end{array}$} & \multirow{2}{*}{$\begin{array}{l}\text { Total } \\
\text { Operating Cost } \\
\text { (\$) }\end{array}$} \\
\hline & G1 & G2 & G3 & G4 & G5 & G6 & G7 & G8 & G9 & & & \\
\hline 1 & 0 & 0 & 0 & 60.40 & 50 & 50 & 100.00 & 0 & 60.00 & 5486.00 & 100 & 5586.00 \\
\hline 2 & 0 & 0 & 0 & 55.00 & 50 & 50 & 95.30 & 0 & 60.00 & 5329.90 & 0 & 5329.90 \\
\hline 3 & 0 & 0 & 0 & 55.00 & 50 & 50 & 88.91 & 0 & 58.30 & 5223.60 & 0 & 5223.60 \\
\hline 4 & 0 & 0 & 0 & 55.00 & 50 & 50 & 89.20 & 0 & 60.00 & 5259.50 & 0 & 5259.50 \\
\hline 5 & 0 & 0 & 0 & 63.40 & 50 & 50 & 100.00 & 0 & 60.00 & 5542.50 & 0 & 5542.50 \\
\hline 6 & 0 & 0 & 0 & 76.40 & 50 & 50 & 100.00 & 10.00 & 60.00 & 6270.30 & 95 & 6365.30 \\
\hline 7 & 0 & 0 & 0 & 57.60 & 50 & 50 & 100.00 & 45.70 & 60.00 & 6625.20 & 0 & 6625.20 \\
\hline 8 & 0 & 0 & 0 & 63.40 & 50 & 50 & 100.00 & 50.00 & 60.00 & 6821.80 & 0 & 6821.80 \\
\hline 9 & 0 & 0 & 0 & 69.40 & 50 & 50 & 100.00 & 50.00 & 60.00 & 6935.20 & 0 & 6935.20 \\
\hline 10 & 0 & 0 & 0 & 76.40 & 50 & 50 & 100.00 & 50.00 & 60.00 & 7068.10 & 0 & 7068.10 \\
\hline 11 & 0 & 0 & 0 & 71.40 & 50 & 50 & 100.00 & 50.00 & 60.00 & 6973.10 & 0 & 6973.10 \\
\hline 12 & 0 & 0 & 0 & 67.40 & 50 & 50 & 100.00 & 50.00 & 60.00 & 6897.30 & 0 & 6897.30 \\
\hline 13 & 0 & 0 & 0 & 59.40 & 50 & 50 & 100.00 & 50.00 & 60.00 & 6746.40 & 0 & 6746.40 \\
\hline 14 & 0 & 0 & 0 & 56.00 & 50 & 50 & 100.00 & 42.30 & 60.00 & 6526.30 & 0 & 6526.30 \\
\hline 15 & 0 & 0 & 0 & 56.60 & 50 & 50 & 100.00 & 37.70 & 60.00 & 6444.90 & 0 & 6444.90 \\
\hline 16 & 0 & 0 & 0 & 56.60 & 50 & 50 & 100.00 & 34.70 & 60.00 & 6384.60 & 0 & 6384.60 \\
\hline 17 & 0 & 0 & 0 & 56.60 & 50 & 50 & 100.00 & 39.70 & 60.00 & 6485.10 & 0 & 6485.10 \\
\hline 18 & 0 & 0 & 0 & 57.60 & 50 & 50 & 100.00 & 46.70 & 60.00 & 6645.40 & 0 & 6645.40 \\
\hline 19 & 0 & 0 & 0 & 69.70 & 50 & 50 & 100.00 & 43.70 & 60.00 & 6813.10 & 0 & 6813.10 \\
\hline 20 & 0 & 0 & 0 & 60.00 & 50 & 50 & 100.00 & 39.30 & 60.00 & 6541.00 & 0 & 65410 \\
\hline 21 & 0 & 0 & 0 & 56.00 & 50 & 50 & 100.00 & 31.30 & 60.00 & 6305.40 & 0 & 6305.40 \\
\hline 22 & 0 & 0 & 0 & 80.00 & 50 & 50 & 100.00 & 48.40 & 10.00 & 6164.30 & 0 & 6164.30 \\
\hline 23 & 0 & 0 & 0 & 78.40 & 50 & 50 & 100.00 & 50 & 0 & 5752.70 & 0 & 5752.70 \\
\hline 24 & 0 & 0 & 0 & 74.40 & 50 & 50 & 100.00 & 50 & 0 & 5676.50 & 0 & 5676.5 .00 \\
\hline \multicolumn{10}{|c|}{ Total Fuel Cost (\$) } & 150918.20 & 195 & 151113.20 \\
\hline
\end{tabular}

Table 6. Comparison of execution time, speedup factor and cluster efficiencies of IEEE 30 bus system for SCUC

\begin{tabular}{|c|c|c|c|c|c|c|c|c|c|c|c|}
\hline No of Processors & 1 & 2 & 4 & 6 & 8 & 10 & 12 & 14 & 16 & 18 & 20 \\
\hline Execution time (s) & 1562.34 & 929.76 & 551.94 & 394.44 & 300.38 & 251.52 & 213.67 & 190.48 & 169.04 & 153.43 & 141.74 \\
\hline Speedup factor & -- & 1.68 & 2.83 & 3.96 & 5.2 & 6.21 & 7.31 & 8.2 & 9.24 & 10.18 & 11.02 \\
\hline Cluster efficiency (\%) & -- & 84 & 70.75 & 66 & 65 & 62.1 & 60.91 & 58.57 & 57.75 & 56.55 & 55.11 \\
\hline
\end{tabular}

\section{Conclusion}

This paper presents a parallel hybrid EIGA algorithm for security constraint unit commitment using distributed memory model. The constraints minimum up/down, ramp up/down, line flows and bus voltages are considered for simulation. The proposed approach utilizes the global and local exploration capabilities of parallel hybrid EIGA to search for the optimal setting of the state variables by considering security constraints into account. Two test systems (Standard 6 bus system and IEEE 30 bus system) are used for testing the superiority of the proposed method. The method has been shows better results than the other methods, which are available in the literature. The performance analysis is carried out based on the speedup factor with varying cluster sizes. The parallel approach is not only reduced the execution time but also improved the quality of solution. To economies the optimum use of available personal computers or workstations, distributed clusters are found to be an alternative viable option. 


\section{References}

[1] Fu Y., Shahidehpour M. \& Li Z. Security-constrained unit commitment with AC constraints, IEEE Trans. Power Syst. 2005; 20: 1538-1550. http://dx.doi.org/10.1109/TPWRS.2005.854375

[2] Fu Y., Shahidehpour M. \& Li Z. C contingency dispatch based on security-constrained unit commitment, IEEE Trans. Power Syst. 2006; 21: 897-908. http://dx.doi.org/10.1109/TPWRS.2006.873407

[3] Hosam K. Youssef \& Khaled M. El-Naggar. Genetic based algorithm for security constrained power system economic dispatch, Electric Power System Research. 2000; 53: 47-51. http://dx.doi.org/10.1016/S0378-7796(99)00039-5

[4] Chandler W.G., Dandeno P.L., Gilmn A.F. \& Kirchmayer L.K. Short-Range Operation of a Combined Thermal and Hydroelectric Power System, AIEE, Transactions. 1953 Oct; 53-326. PMid:13179254

[5] Pang C.K \& Chen H.C. Optimal short-term thermal unit commitment, IEEE Transactions on Power Apparatus and Systems, PAS. 1976; 95(4): 1336-1342. http://dx.doi.org/10.1109/T-PAS.1976.32228

[6] Pang C.K., Sheble, G.B \& Albuyeh F. Evaluation of dynamic programming based methods and multiple area representation for thermal unit commitments, IEEE Transactions on Power Apparatus and Systems PAS. 1996; 100(3): 1212-1218. http://dx.doi.org/10.1109/TPAS.1981.316592

[7] Bertsekas D.P., Lauer, N.R G.S., Sandell Jr. \& Posbergh T.A. Optimal short-term scheduling of large-scale power systems, IEEE Transactions on Automatic Control AC. 1983; 28(1): 1-11. http://dx.doi.org/10.1109/TAC.1983.1103136

[8] Cohen A \& Sherkat V. Optimization-based methods for operations scheduling, Proc. IEEE. 1987; 75 (12): $1574-1591$. http://dx.doi.org/10.1109/PROC.1987.13928

[9] Lei Wu, M. Shahidehpour \& Tao Li. Stochastic Security-Constrained Unit Commitment, IEEE Transactions on Power Systems. 2007; 22 (2): 800-811. http://dx.doi.org/10.1109/TPWRS.2007.894843

[10] Jianhui Wang, Mohammad Shahidehpour \& Zuyi Li. Security-Constrained Unit Commitment With Volatile Wind Power Generation, IEEE Transactions on Power Systems. 2008; 23 (3): 1319-1326. http://dx.doi.org/10.1109/TPWRS.2008.926719

[11] Rolf Hempel \& David W. Walker. The emergence of the MPI message passing standard for parallel computing, Computer standards \& Interfaces. 1999; 21: 51-62. http://dx.doi.org/10.1016/S0920-5489(99)00004-5

[12] Fung C. C, Chow S.Y. \& Wong K.P. A low-cost parallel computing platform for power engineering applications, International conference on APSCOM. 2000; 2: 354-358.

[13] Ghassan Fadlallah, Michel Lavoie \& Louis A. Dessaint. Parallel computing environment and methods, International conference on Parallel computing in Electrical Engineering. 2000; 2-7.

[14] Umpei Nagashima, Sachiko Hyugaaji, Satoshi Sekiguchi, Mitsuhisa Sato \& Haruo Hosoya. An experience with super-linear speedup achieved by parallel computing on a workstation cluster: Parallel calculation of density of states of large scale cyclic polyacenes, Parallel computing. 1995; 21: 1491-1504. http://dx.doi.org/10.1016/0167-8191(95)00026-K

[15] Dasgupta D \& McGregor. Thermal unit commitment using genetic algorithm, IEEE proceedings Gener. Trans. and Distr. 1994; 141(5): 459-465.

[16] Swarup K. S \& Yamashira. S. Unit commitment solution methodology using genetic algorithm, IEEE Trnacactions on Power systems. 2002; 17(1): 87-91.

[17] Senjyu. T, Yamashiro. H, Shimabukuro. K, Uezato. K \& Funabashi. T. Fast solution technique for large-scale unit commitment problem using genetic algorithm, IEEE proceedings Gener. Trans. and Distr. 2003; 150(6): 753-760.

[18] K Chandrasekaran \& Sishaj P Simon. An Enhanced Inherited Crossover GA for the Reliability Constrained UC Problem, Proc. of Int. Joint Colloquium on Emerging Technologies in Computer Electrical and Mechanical. 2011; 76-84.

[19] Bai X \& Wei, H. Semi-definite programming-based method for security constrained unit commitment with operational and optimal power flow constraints, IET Gener. Trans. and Distr. 2009; 3(2): 182-197. http://dx.doi.org/10.1049/iet-gtd:20070516

[20] Ali Daneshi, Mojtaba Khederzadeh, Nima Sadrmomtazi \& Javad Olamaei. Integration of Wind power and Energy Storage in SCUC Problem, World Non Grid-Connected Wind Power and Energy Conference (WNWEC). 2010; 1-8. http://dx.doi.org/10.1109/WNWEC.2010.5673246 